\title{
Lung transplant with bronchial arterial revascularization: review of surgical technique and clinical outcomes
}

\author{
James J. Yun, Shinya Unai, Gosta Pettersson \\ Department of Thoracic and Cardiovascular Surgery, Cleveland Clinic, Cleveland, OH, USA \\ Contributions: (I) Conception and design: All authors; (II) Administrative support: G Pettersson; (III) Provision of study materials or patients: G \\ Pettersson; (IV) Collection and assembly of data: JJ Yun, G Pettersson; (V) Data analysis and interpretation: All authors; (VI) Manuscript writing: All \\ authors; (VII) Final approval of manuscript: All authors. \\ Correspondence to: James J. Yun, MD, PhD. Cleveland Clinic, J-4 9500 Euclid Avenue, Cleveland, OH 44195, USA. Email: yunj@ccf.org.
}

\begin{abstract}
Lung transplantation (LTx) is routinely performed with sacrifice of the bronchial arterial circulation. We review bronchial artery anatomy and surgical technique for LTx with direct bronchial arterial revascularization (BAR). We also update the published clinical experience, including from our center, of LTx with BAR. Published series of LTx with BAR are from single centers, and all cite high technical success rates and good short-term outcomes. Technically, double LTx (DLTx) with BAR is almost always possible if a deliberate review of bronchial artery anatomy is performed. For single LTx (SLTx), BAR is feasible in about $50 \%$ of cases. The combined Copenhagen and Cleveland Clinic experience (with BAR procedures performed or supervised by Dr. Gosta B. Pettersson) includes 131 LTx with BAR with a technical success rate $>95 \%$. Procedural success is uniformly associated with normal airway healing. Five-year survival in LTx with BAR is superior to the 5-year survival of LTx patients in the ISHLT registry. LTx with BAR is feasible and safe, and technical success ensures normal airway healing. The experience with BAR at experienced centers suggests possible long-term survival benefit. A multicenter study is needed to define the role of BAR in LTx.
\end{abstract}

Keywords: Lung transplantation (LTx); bronchial arteries; bronchial arterial revascularization (BAR)

Submitted Apr 16, 2019. Accepted for publication Aug 30, 2019.

doi: $10.21037 /$ jtd.2019.09.09

View this article at: http://dx.doi.org/10.21037/jtd.2019.09.09

\section{Introduction}

The bronchi have a dual blood supply from (I) bronchial arteries, which originate from the aorta, and (II) pulmonary arterial circulation. Experimental lung transplantation (LTx) with bronchial arterial revascularization (BAR) is credited to Metras in the 1950s and Haglin in the 1970s $(1,2)$. Successful BAR improved early airway healing, and decreased airway complications $(1,2)$.

In the 1990s, several lung transplant centers reported successful clinical series of LTx with BAR. Couraud and colleagues (Bourdeaux, FR) reported double LTx (DLTx) with BAR ( $\mathrm{n}=8)$ with saphenous vein graft conduit in 1992. The authors noted that LTx with BAR increased the length of surgery by $\sim 40$ minutes, but reduced ischemia time $v$. bilateral sequential LTx (3). Dr. Yacoub and colleagues
(Harefield Hospital, UK) reported 9 successful DLTx with BAR using internal mammary artery conduit. There were no airway complications and 2 reoperations for bleeding. Two mortalities were related to severe graft dysfunction and intracranial hemorrhage (4). Daly and McGregor (Mayo Clinic, USA) reported outcomes of 10 single LTx (SLTx) with BAR in 1994. In 9 survivors, 7 had patent BAR on postoperative angiogram (5).

At our institution, Dr. Gosta B. Pettersson introduced LTx with BAR to Cleveland Clinic after his Copenhagen experience, which included 106 LTx with BAR associated with a procedural success rate $>95 \%$ and excellent airway healing in all technically successful cases. The survival of patients with DLTx and BAR in the Copenhagen experience ( $80 \%$ survival at 5 years) significantly exceeded the 5 -year survival of LTx patients in the ISHLT registry ( 50\%) (6). 


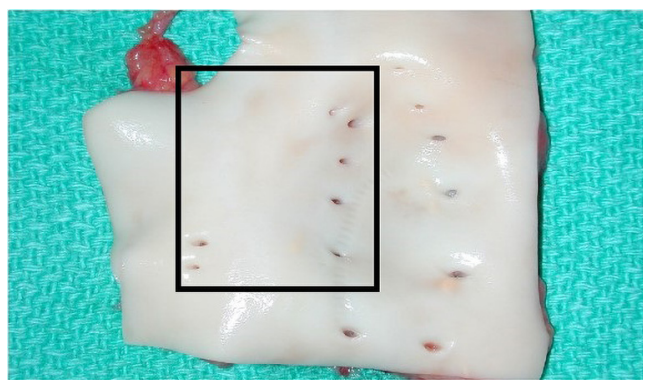

Figure 1 The location of the bronchial artery orifices in the descending thoracic aorta of the donor. The rectangle denotes the area in which left and right bronchial arteries (or an intercostobronchial artery trunk supplying the right side) are typically found.

The modern technique of bilateral sequential LTx (without BAR) is associated with airway complication rates as high as $25 \%$. The lack of surgical enthusiasm for LTx with BAR in the broader LTx community is due to multiple factors, including increased procedural complexity, concerns regarding ischemia time, and limited opportunities for surgeons to gain experience in the identification and handling of the bronchial arteries. Moreover, there is concern about the consequence of BAR thrombosis or stenosis on the lung bloc.

In this report, we review bronchial artery anatomy, surgical technique of SLTx and DLTx with BAR, and review recent clinical results of LTx with BAR.

\section{Bronchial artery anatomy}

Anatomic human bronchial artery variations from 30 autopsies were described by Schreinemakers et al. (7). In brief, bronchial arteries originate from the upper descending aorta (Figure 1), and the right mainstem bronchus is typically supplied by the right intercostobronchial artery (RICBA). The RICBA originates as the first or second right intercostal artery and gives of an intercostal branch after coursing behind the esophagus. It then passes under the azygos vein before supplying the membranous portion of the right main bronchus (Figure 2). Bronchial artery pedicle origin from the RICBA was feasible in $17 / 19$ cases. Our group has found the presence and course of the RICBA more variable. On the left, a single left bronchial artery supplying the left mainstem bronchus is usually noted, but care should be taken to rule out the presence of additional left bronchial arteries.

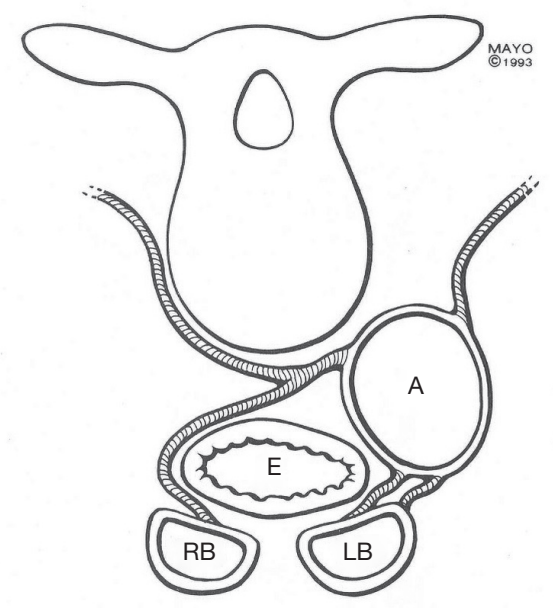

Figure 2 Schematic drawing of the typical course of the left bronchial artery and right retroesophageal intercostobronchial artery. A, aorta; E, esophagus; RB, right mainstem bronchus; LB, left mainstem bronchus.

Intrapulmonary bronchial artery capillaries drain into pulmonary veins $(8,9)$, while extrapulmonary bronchial capillaries drain into the azygos and hemiazygos veins (10). Since bronchial arteries drain into the pulmonary veins, bleeding risk from LTx with BAR is not related to venous drainage.

\section{BAR: donor operation}

\section{Procurement}

The donor procurement operation is based on the technique described by Schreinemakers et al. (7). After intravenous (IV) heparin is administered, the donor aorta is cross clamped and cold lung perfusate is administered via the pulmonary artery (PA). The lungs are removed en bloc with the trachea in standard fashion, with the accompanying thoracic esophagus and descending aorta. The RICBA and left bronchial artery (or arteries) are therefore included. The trachea and esophagus are stapled and divided with care taken to stay above the azygos vein. Inferiorly, the aorta is divided at the level of the diaphragm. Finally, the double lung bloc with accompanying tissue is excised from the chest moving cranially, including paraspinal tissue to minimize the possibility of injury to the RICBA and left bronchial arteries. The main PA is divided near the PA bifurcation if the heart is taken separately for transplant. Care is taken to leave sufficient left atrial cuff in standard fashion. 


\section{Lung bloc preparation (DLTx)}

After arrival in the recipient operating room (OR), the esophagus is removed. With the lung bloc upright, cranial traction of the esophagus facilitates careful sharp dissection from surrounding mediastinal tissue. As progress is made caudally, care is taken to stay close to the esophageal adventitial layer to avoid bronchial artery injury. After the esophagus is removed, mediastinal tissue including the bronchial arteries remains. Attention is then turned to the descending aorta, which is opened vertically in the midline on its pleural-covered surface. Orifices of the bronchial and intercostal arteries are then visible as shown (Figure 1). Knowledge of the typical RICBA and left bronchial artery locations is important in proper vessel identification (Figure 1, inset rectangle). Selective use of coronary probes can facilitate proper identification of the bronchial arteries and their course. On the right, once the RICBA is identified, clips are applied to its intercostal branch. The left bronchial artery (or arteries) are identified with probing if needed.

On either side, a single bronchial artery of reasonable size is usually sufficient for complete revascularization. However, if a convincing bronchial artery is not identified or if an important bronchial artery is damaged, BAR should not be aborted and standard bilateral sequential LTx with distal bronchial anastomoses should be performed. The trachea is sharply divided one ring above the carina. Pericarinal tissue is left intact to avoid damage to the bronchial arteries. Only excess tissue that clearly lacks bronchial arteries is excised.

\section{SLTx with BAR}

When right and left lungs are allocated to different centers, the need for lung bloc division at the donor hospital is an impediment to BAR. Bronchial artery course defines proper bloc division. Careful division and excision of peribronchial tissue with awareness of bronchial artery course are required to avoid injury. Right SLTx with BAR is performed only if a reasonably sized RICBA is present, and properly preserved. In our experience, revascularization of both a right single and left single lung with BAR is feasible in less than half of cases. For SLTx with BAR, the bronchus is trimmed back as usual to just above the secondary carina before implantation, paying attention to leaving peribronchial mediastinal tissue around the airway. Bronchial arteries coursing centrally are at risk when the bronchial anastomosis is constructed. However, with distal bronchial anastomosis, the consequences of BAR failure are minimal.

\section{Revascularization and conduit options}

Couraud et al. reported successful BAR with saphenous vein conduit (3). Our preference is to utilize the internal thoracic artery (ITA), as described by Sir Magdi Yacoub (Harefield Hospital, UK) (4). The pediatric lung transplant group at Texas Children's Hospital (Houston, TX, USA) has successfully used donor aortic patch containing the origin of both bronchial arteries, sewn directly to the recipient aorta (11).

\section{Recipient operation: en bloc DLTx with BAR}

En bloc DLTx with BAR is performed via sternotomy. The left internal mammary artery (LIMA) is harvested in standard fashion and left in continuity until after systemic heparinization. Full cardiopulmonary bypass is used. We favor ascending aortic and bicaval cannulation and placement of a PA vent. Recipient lungs are excised as for bilateral sequential lung transplant; both mainstem bronchi are stapled. When a PA vent is used, the pulmonary arteries can simply be transected. The trachea is then resected to 1 ring above the carina, after the space between, and posterior to, the ascending aorta and superior vena cava is opened. The right and left pulmonary arteries are resected, to the main PA trunk.

The donor lungs are then carefully introduced into their respective pleural spaces, posterior to the heart and through the respective pericardial openings, with particular attention paid to maintain proper orientation. Since the pericardial openings leading to the pleural spaces are limited in size (particularly on the right), attention must be paid to avoid torsion.

The tracheal anastomosis is performed with running 3-0 or 4-0 prolene, with peritracheal tissue used to cover the anastomosis. Attention is then turned to the LIMA to bronchial artery anastamosis. Retraction of the left lung anteriorly and to the right exposes the proximal descending aorta and bronchial artery origins. An end-side anastomosis between the LIMA and donor aorta and bronchial artery orifices (with sequential anastomoses performed if needed) is constructed with running 7-0 prolene. Flow to the LIMA is established. Successful bronchial artery revascularization is immediately confirmed by observing bleeding from 
donor mediastinal tissue and the tracheal anastomosis. If no bleeding is identified, flow in the internal mammary artery (IMA) can also be assessed with a flow probe (as used for graft assessment in coronary artery bypass grafting). Airway healing is possible with perfusion of 1 bronchial artery if it is of sufficient size; airway healing can also occur without visualization of circumferential bleeding around the trachea.

At this point the IMA can be temporarily occluded with a bulldog, or left open. Bronchial artery reperfusion allows identification of potential bleeders, but rewarms the lungs earlier than usual. It remains to be determined if reperfusion at this stage is beneficial, although theoretically this should be the case.

The remaining anastomoses are then performed. The main PA anastomosis (at main PA above the pulmonary valve) and atrial cuff anastomoses bilaterally are constructed with running prolene suture.

Overall, performing en bloc DLTX with BAR does not add significantly to ischemic time $v s$. standard bilateral sequential LTx.

\section{Recipient operation: SLTx with BAR}

The IMA is harvested as for DLTx with BAR. SLTx is performed in the usual fashion (as in SLTx without BAR). Low dose IV heparin (5,000 units) is given before the lung is reperfused, The lung is ventilated before bronchial revascularization is performed. The IMA is divided and anastomosed to the selected bronchial artery orifice(s) in the donor descending aorta. These arteries commonly present themselves via back bleeding following PA reperfusion. Since the bronchial anastomosis in SLTx with BAR is performed last, ischemic time is not prolonged.

\section{Lung transplant with BAR: early experience}

Haglin et al. reported left SLTx with BAR using the technique described by Metras $(1,2)$. Couraud and coworkers (3) published the first successful series of DLTx with BAR in seven patients, using saphenous vein conduit. Successful BAR was confirmed with early bronchoscopy and arteriography. At 2 weeks postop, vein grafts were patent in 5/7 LTx recipients. The same group reported three additional BAR cases (2 DLTx and 1 right SLTx) in 1991, with good airway healing and patent vein grafts in two cases studied angiographically (12). Baudet et al. studied 15 patients who underwent LTx with BAR, with follow-up of 22-69 months (13). In five cases with failed BAR, all had clinical bronchiolitis obliterans syndrome (BOS) and 4 had histologic evidence of obliterative bronchiolitis (OB). In contrast, none of the seven patients with functioning BAR had clinical BOS or OB. The authors suggested that BAR might be protective against BOS/OB.

Daly and co-workers published results of nine cases of DLTx with BAR in 1993 (4). The largest vessel going in the direction of the carina was chosen for revascularization with the left IMA. Bronchoscopy and arteriography showed patient IMA-bronchial artery anastomoses and normal airway healing in $7 / 8$ cases. The case of failed BAR was associated with airway ischemia, and airway ulceration at the tracheal anastomosis.

In 1994 Daly and McGregor (5) published the results of 10 SLTx with BAR at Mayo Clinic, Rochester. In 9 surgical survivors, angiography demonstrated patent BAR in 7 and occluded BAR in 2.

\section{Lung transplant with BAR: Copenhagen experience}

Dr. Gosta B. Pettersson reported the initial Copenhagen BAR experience in 1994 (14). This series included 68 DLTx with BAR, 1 bilateral sequential lung $\mathrm{Tx}_{\mathrm{x}}$ with right lung BAR only (due to bronchial anatomy), 27 SLTx with BAR, and 10 HLTx (15-20).

Early (3 month) mortality for en bloc DLTX was $10.2 \%$ $(16,17)$. Overall 5- and 10-year survival for DLTX with BAR were $63 \%$ and $36 \%$, respectively (5).

In the first 47 DLTx BAR cases, reoperation for bleeding was necessary in 5 (15). The source of bleeding was the LIMA-bronchial artery anastomosis in three cases, all of which occurred in the early experience. In these cases, multiple bronchial orifices from the descending aorta were covered with a single IMA. This technique was revised in favor of use of sequential anastomoses when bronchial artery orifices were separated by more than $5 \mathrm{~mm}$. In one case, additional stitch placement for hemostasis resulted in BAR occlusion on postop angiogram, which was rectified at reoperation.

In 53 cases of LTx with BAR, angiography confirmed success in 50 cases, with 3 technical failures (18). In the three cases of failed BAR, airway ischemia and impaired bronchial healing were confirmed bronchoscopically. In 2 DLTx cases with failed BAR, mucosal necrosis preceded stenosis of the left mainstem bronchus. We suggest that in cases of en bloc DLTx with failed BAR, the tracheal suture line had sufficient ability to heal from peritracheal tissue, 

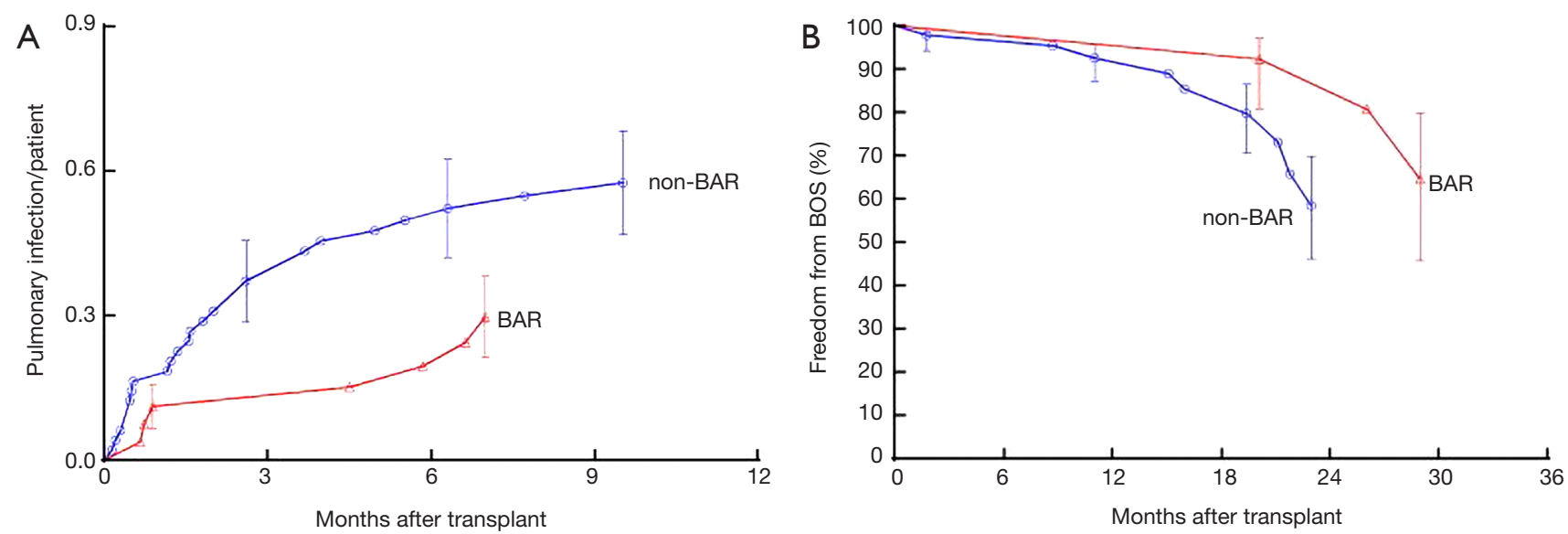

Figure 3 Results of lung transplant with BAR at Cleveland Clinic. (A) Cumulative incidence of pulmonary infections during the first year after transplantation among propensity-matched BAR and non-BAR patients. Symbols are nonparametric estimates, and vertical bars are $68 \%$ confidence limits equivalent to \pm 1 SEM. The vertical axis is cumulative incidence, and number of patients remaining at risk is greater because patients remain at risk of infections (21); (B) freedom from BOS after lung transplant for BAR and propensity-matched non-BAR patients. Symbols are nonparametric estimates, and vertical bars are $68 \%$ confidence limits equivalent to \pm 1 SEM (21). BAR, bronchial arterial revascularization; SEM, standard error of mean; BOS, bronchiolitis obliterans syndrome.

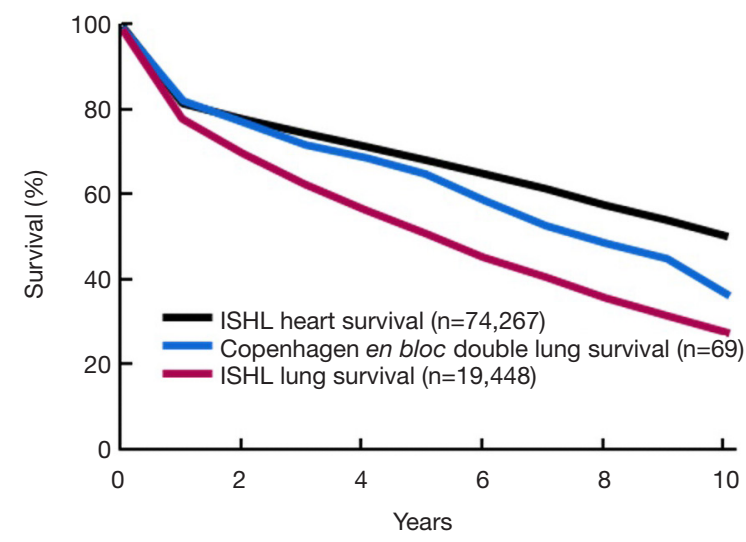

Figure 4 Actuarial survival after heart transplantation (2008 ISHLT data), lung transplantation (2008 ISHLT data), and Copenhagen DLTx with BAR $(n=69)$. BAR, bronchial arterial revascularization; DLTx, double lung transplantation.

but that distal ischemia manifested as bronchial mucosal necrosis. In both cases the patients survived but required left pneumonectomy. The $3 \mathrm{rd}$ case of failed BAR was a patient who required en bloc DLTx with BAR after failed single left lung transplant (non-BAR); cause of death was sepsis related to pulmonary Pseudomonas and Aspergillus 30 days post-transplant.

In the Copenhagen series, the incidence of clinical BOS was $28.3 \%(15 / 53)$ in patients who underwent DLTx with
BAR, at a mean of $18 \pm 11$ months postoperatively, with mean follow-up of 31 months. BOS was histologically confirmed in seven patients. One patient developed OB 25 months post-transplant without meeting clinical BOS criteria.

No internal control group was available for the 38 patients in the Copenhagen series, but comparison of results with those of Stanford University (26 patients who underwent LTx without BAR) suggested a trend towards freedom from OB and BOS in Copenhagen (Figure 3). Clearly, the sample size in both groups was small and studies with larger numbers of patients are needed to draw firmer conclusions.

Figure 4 shows the Kaplan-Meier survival plots for Copenhagen DLTX with BAR $(\mathrm{n}=69)$ versus the ISHLT survival data for LTx $(\mathrm{n}=19,488)$ and ISHLT heart transplant (HTx) $(\mathrm{n}=74,267)$.

We speculate that a potential mechanism by which BAR could inhibit $\mathrm{OB}$ and BOS, apart from improvement in early postop airway healing, is facilitating direct vascular allograft entry for immune mediators such as helper $\mathrm{T}$ cells or anti-inflammatory cytokines such as IL-10 that would inhibit development of chronic rejection.

\section{Lung transplant with BAR: Cleveland experience}

At Cleveland Clinic, Pettersson and colleagues reported results of a propensity-matched study of LTx with BAR $v$ s. 


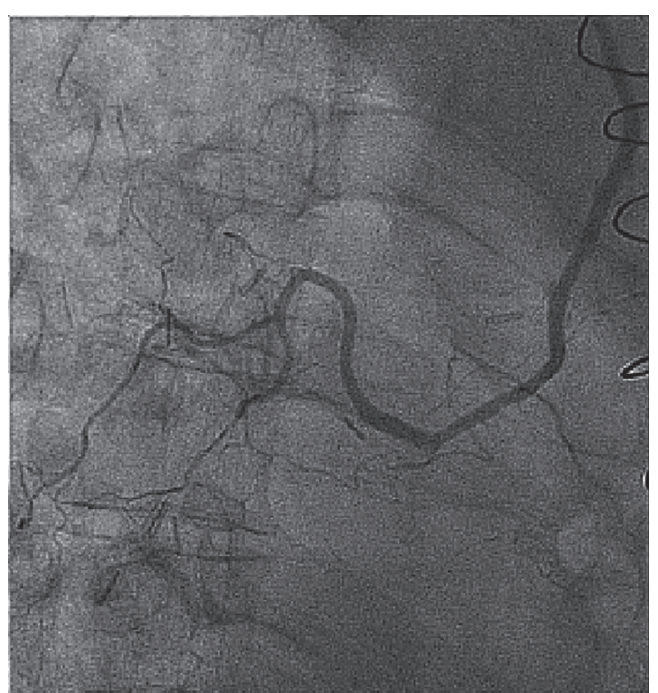

Figure 5 Successful BAR documented on angiogram after en bloc DLTx. BAR, bronchial arterial revascularization; DLTx, double lung transplantation.

LTx without BAR (21). Twenty-seven patients underwent LTx with BAR (20 DLTx and 7 SLTx) with mean lung allocation score (LAS) 46 4 16. LTx indications were idiopathic pulmonary fibrosis/usual interstitial pneumonia (UIP) ( $n=12)$, cystic fibrosis $(n=4)$, chronic obstructive pulmonary disease (COPD) $(\mathrm{n}=3)$, pulmonary hypertension $(\mathrm{HTN})(\mathrm{n}=2)$, sarcoid $(\mathrm{n}=1)$, non-specific interstitial pneumonia (NSIP) ( $\mathrm{n}=1$ ), Sjögren's-related interstitial lung disease (ILD) ( $n=2)$, eosinophilic granuloma $(n=1)$, and post-radiation bronchiolitis $(\mathrm{n}=1)$.

LTx with BAR was successful in $26 / 27$ cases, with normal airway healing in all but 1 case of failed BAR (2.4\%). Arteriography confirmed successful BAR in $96 \%$ of patients, including one with poor success. An example of arteriography after successful DLTx is shown (Figure 5). The single case of failed BAR after DLTx resulted in central airway (distal tracheal) ischemia with mucosal sloughing and areas of deep necrosis. Remarkably, the patient did not develop any airway obstruction and the tracheal anastomosis eventually healed well without any intervention. Overall, no BAR patients required airway intervention.

BAR and non-BAR patients had similar operative (skinskin) times and overall hospital length of stay (LOS), but BAR patients required more reoperations for bleeding $(\mathrm{P}<0.002)$. Reoperations for anastomotic bleeding were mainly related to the LIMA-bronchial artery anastomosis. In most cases at reoperation the solution to bleeding was tightening of the suture at this anastomosis. Usually this can be accomplished without need for full CPB, by using a bronchial blocker and selective (right) lung ventilation.

One early (in-hospital) death occurred after BAR, vs. 2/54 in the non-BAR group. Postoperative median intubation time was 1.5 days, ICU stay 5.0 days, and hospital stay 18 days. No patients transplanted with BAR to our knowledge have yet developed BOS or OB, with the longest follow-up 29 months. Overall survival was $92 \%$ (23/25) with a mean follow-up of 7 months. Two late deaths were noted; the first resulted from metastatic cancer in the native (non-transplanted) lung 16 months after SLTx with BAR. The second death was a sudden death 22 months post-transplant unrelated to OB or BOS.

\section{Texas Children's Hospital experience}

The lung transplant program at Texas Children's Hospital led by Dr. Emmett McKenzie has reported 31 pediatric lung transplants with BAR from 2005-2014, compared with 88 bilateral sequential lung transplants (BSLTx) performed without BAR during the same period (11). Recipient age ranged from 2 months-20 years. Surgical technique was notable for use of en bloc DLTx with a donor aortic button (containing both bronchial artery origins) sewn to recipient descending donor thoracic aorta for bronchial revascularization. The authors reported a superior overall survival rate for en bloc DLTx with BAR vs. bilateral sequential LTx (77\% vs. 61\%). Freedom from BOS was also superior for en bloc DLTx with BAR $v s$. BSLTx without BAR (94\% vs. 71\%). Ischemic airway findings on bronchoscopy were also less frequent in the BAR group (16\% vs. $57 \%$ in bilateral sequential LTx without BAR).

In this series ischemia time was not higher for transplants with BAR, and no en bloc DLTx with BAR patients required reoperation for bleeding.

\section{Generalizability of lung transplant with BAR}

Over the past 20 years, adoption of LTx with BAR has been limited to very few centers worldwide, despite the procedural benefits of reduced airway ischemia and decreased rejection (22). Barriers to greater adoption of LTx with BAR include perceived technical difficulty and prolongation of operative time. In addition, lack of general recognition of the impact of airway ischemia on outcomes and postop complications after LTx results in absence of demand for BAR. Only multicenter collaboration between 
believers in BAR can change this!

In the future, we hope that utilization of simulation, $3 \mathrm{D}$ printing, and ex vivo lung perfusion will permit opportunities for (I) increased understanding of the nuances of human bronchial artery anatomy, and (II) additional opportunities for identification and safe dissection of bronchial arteries to mitigate the learning curve associated with BAR.

\section{Conclusions}

Lung transplant with BAR is feasible and safe. Successful BAR is associated with minimal airway ischemia, and BAR can be performed with a success rate $>90 \%$. In our view the benefits outweigh the risks. A multicenter study of BAR is required to further define the role of BAR in LTx.

\section{Acknowledgments}

None.

\section{Footnote}

Conflicts of Interest: The authors have no conflicts of interest to declare.

Ethical Statement: The authors are accountable for all aspects of the work in ensuring that questions related to the accuracy or integrity of any part of the work are appropriately investigated and resolved.

\section{References}

1. Haglin JJ, Ruiz E, Baker RC, et al. Histologic studies of human lung allotransplantation. In: Wildevuur C. editor. Morphology in lung transplantation. Basel: S. Karger, 1973:13-22.

2. Metras H. Note préliminaire sur la greffe totale du poumon chez le chien. C R Acad Sci (Paris) 1950;231:1176-8.

3. Couraud L, Baudet E, Martigne C, et al. Bronchial revascularization in double-lung transplantation: a series of 8 patients. Bordeaux lung and heart-lung transplant group. Ann Thorac Surg 1992;53:88-94.

4. Daly RC, Tadjkarimi S, Khaghani A, et al. Successful double-lung transplantation with direct bronchial artery revascularization. Ann Thorac Surg 1993;56:885-92.

5. Daly RC, McGregor CG. Routine immediate direct bronchial artery revascularization for single-lung transplantation. Ann Thorac Surg 1994;57:1446-52.

6. Burton CM, Milman N, Carlsen J, et al. The Copenhagen National Lung Transplant Group: survival after single lung, double lung, and heart-lung transplantation. J Heart Lung Transplant 2005;24:1834-43.

7. Schreinemakers HH, Weder W, Miyoshi S, et al. Direct revascularization of bronchial arteries for lung transplantation: an anatomical study. Ann Thorac Surg 1990;49:44-53; discussion 53-4.

8. Wagenvoort CA, Wagenvoort N. Arterial anastomoses, bronchopulmonary arteries, and pulmobronchial arteries in perinatal lungs. Lab Invest 1967;16:13-24.

9. Tobin CE. The bronchial arteries and their connections with other vessels in the human lung. Surg Gynecol Obstet 1952;95:741-50.

10. Liebow AA. Patterns of origin and distribution of the major bronchial arteries in man. Am J Anat 1965;117:19-32.

11. Guzman-Pruneda FA, Orr Y, Trost JG, et al. Bronchial artery revascularization and en bloc lung transplant in children. J Heart Lung Transplant 2016;35:122-9.

12. Couraud L, Baudet E, Nashef SAM, et al. Lung transplantation with bronchial revascularization. Surgical anatomy, operative technique and early results. Eur J Cardiothorac Surg 1992;6:490-5.

13. Baudet EM, Dromer C, Dubrez J, et al. Intermediateterm results after en bloc double-lung transplantation with bronchial arterial revascularization. Bordeaux lung and heart-lung transplant group. J Thorac Cardiovasc Surg 1996;112:1292-9; discussion 1299-300.

14. Pettersson G, Arendrup H, Mortensen SA, et al. Early experience of double-lung transplantation with bronchial artery revascularization using mammary artery. Eur J Cardiothorac Surg 1994;8:520-4.

15. Pettersson G, Nørgaard MA, Arendrup H, et al. Direct bronchial artery revascularization and en bloc double lung transplantation--surgical techniques and early outcome. J Heart Lung Transplant 1997;16:320-33.

16. Svendsen U, Arendrup H, Nørgaard M, et al. Double lung transplantation with bronchial artery revascularization using mammary artery. Transplant Proc 1995;27:3485.

17. Nørgaard MA, Olsen PS, Svendsen UG, et al. Revascularization of the bronchial arteries in lung transplantation: an overview. Ann Thorac Surg 1996;62:1215-21.

18. Nørgaard MA, Efsen F, Arendrup H, et al. Surgical and arteriographic results of bronchial artery revascularization 
in lung and heart lung transplantation. J Heart Lung

Transplant 1997;16:302-12.

19. Pettersson G, Nørgaard MA, Andersen CB, et al. Lung and heart-lung transplantation with direct bronchial artery revascularization. In: Hetzer, Reichart. editors. Lung Transplantation. Darmstadt: Dr Dietrich Steinkopf Verlag, 1999.

20. Nørgaard MA, Andersen CB, Pettersson G. Does bronchial artery revascularization influence results concerning bronchiolitis obliterans syndrome and/or

Cite this article as: Yun JJ, Unai S, Pettersson G. Lung transplant with bronchial arterial revascularization: review of surgical technique and clinical outcomes. J Thorac Dis 2019;11(Suppl 14):S1821-S1828. doi: 10.21037/jtd.2019.09.09 obliterative bronchiolitis after lung transplantation? Eur J Cardiothorac Surg 1998;14:311-8.

21. Pettersson GB, Karam K, Thuita L, et al. Comparative study of bronchial artery revascularization in lung transplantation. J Thorac Cardiovasc Surg 2013;146:894900.e3.

22. Tong MZ, Johnston DR, Pettersson GB. The role of bronchial artery revascularization in lung transplantation. Thorac Surg Clin 2015;25:77-85. 